\title{
Optimization of YIG/Bi stacks for spin-to-charge conversion and influence of aging
}

\author{
Soraya Sangiao ${ }^{1,2, *}$, José Ignacio Morales-Aragonés ${ }^{2}$, Irene Lucas ${ }^{1,2}$, Pilar Jiménez- \\ Cavero $^{1,2}$, Luis Morellón ${ }^{1,2}$, Carlos Sánchez-Azqueta ${ }^{4}$, José María De Teresa ${ }^{1,2,3}$ \\ 1/nstituto de Nanociencia y Materiales de Aragón (INMA), CSIC-Universidad de Zaragoza, Zaragoza \\ 50009, Spain \\ 2Departamento de Física de la Materia Condensada, Universidad de Zaragoza, Zaragoza 50009, Spain \\ ${ }^{3}$ Laboratorio de Microscopías Avanzadas (LMA), Universidad de Zaragoza, Zaragoza 50018, Spain \\ ${ }^{4}$ Group of Electronic Design, Aragón Institute of Engineering Research (I3A), University of Zaragoza, \\ Zaragoza 50009, Spain \\ *sangiao@unizar.es
}

\begin{abstract}
We show that an optimized growth of magnetic layer / non-magnetic layer stacks allows for the improvement of the spin-to-charge conversion efficiency. From the analysis of the voltage signal generated in spin pumping experiments due to the inverse spin Hall effect (ISHE) on YIG/Bi stacks, we have determined values for the spin Hall angle and the spindiffusion length in $\mathrm{Bi}$ of $0.0068(8)$ and $17.8(9) \mathrm{nm}$, respectively. Based on these results, we have also studied the influence of aging on the spin-to-charge conversion efficiency by performing spin pumping experiments on YIG/Bi stacks after exposing the samples to ambient conditions for several days, up to 150 days. We have found that in $\mathrm{YIG} / \mathrm{Bi}$ samples with Bi thicknesses around or below the spin-diffusion length, the ISHE voltage signal is still above $80 \%$ of its initial value after 100 days.
\end{abstract}

Keywords: spin pumping, spin Hall effect, yttrium iron garnet, bismuth.

\section{Introduction}

The design of spintronic devices is driven by the ever-increasing demands for high data processing speed, large data storage capability and low power consumption [1-3]. An efficient mutual conversion between spin and charge currents is a key ingredient for the reduction of power consumption in spintronic devices. Spin Hall effect (SHE) has shown its potential for the generation and manipulation of spin-polarized electrical currents [4]. In the inverse SHE (ISHE), a pure spin current is converted into a transversal charge current in a material with large spin-orbit coupling. ISHE has been widely used to electrically detect spin currents since the pioneering works of Saitoh [5] and Valenzuela [6], which indicates the relevance of ISHE for its application in spintronic devices. Experimental studies on spin-to-charge conversion in bismuth, which is one of the most extensively studied elements in solid-state physics due to its very special electronic properties, have shown its feasibility for the efficient conversion of spin currents into charge currents via ISHE [7-10]. 
Regarding the generation of pure spin currents in spin pumping experiments, $\mathrm{Y}_{3} \mathrm{Fe}_{5} \mathrm{O}_{12}$ (YIG) is an ideal candidate due to the combination of electric insulating properties with low dielectric losses and a very narrow ferromagnetic resonance (FMR) linewidth related to a small Gilbert damping $[11,12]$. We report here on the optimization of the growth of YIG/Bi stacks and the study of their spin-to-charge conversion efficiency. YIG thin films have been grown by pulsed laser deposition (PLD) and the characterization of their magnetization dynamics has been performed by means of broadband FMR measurements and subsequent spin pumping experiments [13], once a bismuth layer has been added on top, to be used as an ISHE detector.

Furthermore, the influence of aging on the spin-to-charge conversion efficiency of $\mathrm{YIG} / \mathrm{Bi}$ stacks is studied. To our knowledge, there is no previous study on the time evolution of the ISHE voltage signal in magnetic / non-magnetic stacks while storing the samples in ambient conditions, as it would occur in a practical implementation of these stacks. This phenomenon is important, given that aging could be the cause of the wide discrepancy of spin-to-charge conversion results found in literature for nominally the same materials [14-16]. With this aim in mind, after presenting the frequency dependence of the ISHE voltage signal in YIG/Bi stacks and studying the dependence of the ISHE voltage signal on the $\mathrm{Bi}$ thickness, which enables us to extract all the relevant parameters characterizing the spin-to-charge conversion in these stacks, we have investigated the evolution of the ISHE voltage signal with time.

\section{Methods}

In order to study the spin-to-charge conversion efficiency in YIG/Bi stacks, pure spin currents are injected using the FMR-induced spin pumping from an 85-nm-thick YIG layer epitaxially grown on gadolinium gallium garnet (GGG) substrates of $7 \mathrm{~mm}$ by $2 \mathrm{~mm}$ in size by pulsed laser deposition. YIG thin films were grown at a temperature of $750^{\circ} \mathrm{C}$ in a PLD system using a $\mathrm{KrF}$ excimer laser $(248 \mathrm{~nm})$ at $10 \mathrm{~Hz}$ repetition rate in a 400 mTorr oxygen pressure atmosphere. A stoichiometric polycrystalline YIG target has been used. Samples were then taken to air and introduced into an electron-beam evaporation system, where bismuth layers were grown in vacuum at a base pressure of $10^{-7}$ Torr. Structural characterization by $\mathrm{x}$-ray diffraction (XRD) and ultrahigh-resolution transmission electron microscopy (HRTEM) of $\mathrm{Bi}$ thin films fabricated in the same laboratory and under the same experimental conditions has been previously reported [17], indicating that $\mathrm{Bi}$ films are polycrystalline but strongly textured along the trigonal axis [001] and both, the polycrystalline nature and the texture, remain unmodified when grown on different substrates [18]. Six different YIG/Bi stacks were grown and studied in which the thickness of the Bi layer, determined by $x$-ray reflectivity (XRR), was 9.5, 14.1, $16.9,18.5,28.8$ and $47.1 \mathrm{~nm}$.

FMR and spin pumping measurements were carried out at room temperature on YIG and YIG/Bi samples flipped upside down on a shorted microstrip line placed between the poles of an electromagnet that provides static magnetic fields up to $1 \mathrm{~T}$ in the plane of the samples and perpendicular to the rf field. Excitation frequencies of the of magnetic field in the range from 2 to $15 \mathrm{GHz}$ at a power of $10 \mathrm{dBm}$ have been selected using a Keysight N5173B rf generator. The ISHE voltage signal is measured by a lock-in 
technique with an amplitude modulation at a few $\mathrm{kHz}$, as well as the reflected microwave signal, through an rf circulator and a second lock-in amplifier. A sketch of the experimental configuration used for spin pumping experiments is shown in figure 1.

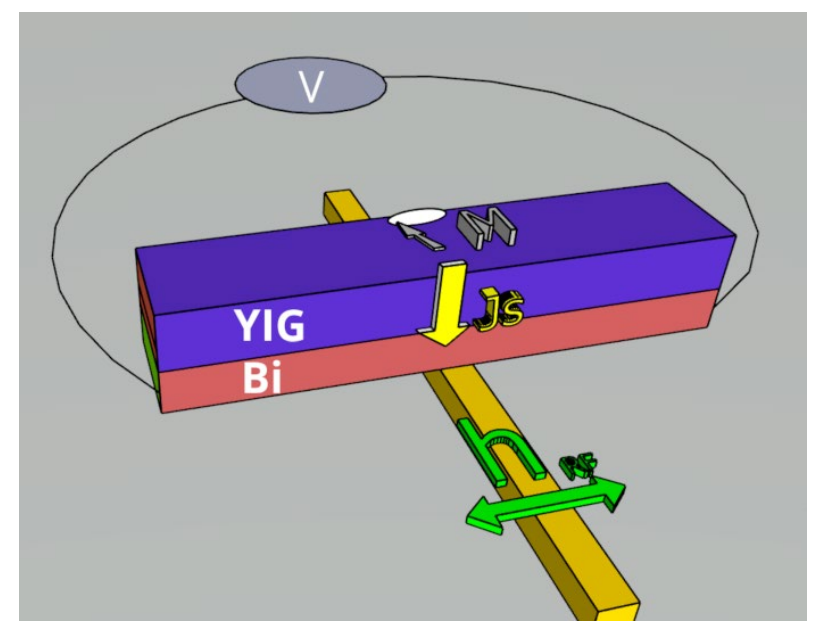

Figure 1. Sketch of the experimental configuration for spin pumping measurements showing the relative orientations of magnetization (parallel to the dc magnetic field), the rf magnetic field and the direction of the spin current injected into the Bi layer from the YIG layer.

\section{Results and discussion}

YIG/Bi stacks were first characterized by XRD. In these experiments, presented in the figure 2(a), an epitaxial growth of the YIG layers is obtained. Indeed, we have observed Laue oscillations, which indicate that YIG films exhibit high crystalline quality and nearly perfect interfaces. The interface quality is a critical parameter to ensure a high efficiency in the transfer of spin-angular momentum across it. To quantitatively determine the thickness of each layer composing the YIG/Bi stacks, XRR has been performed on them. Numerical analysis of the XRR data by means of the commercial software Leptos provided by Bruker allows for the quantitative determination of the thickness of each layer, as illustrated in figure 2(b). 

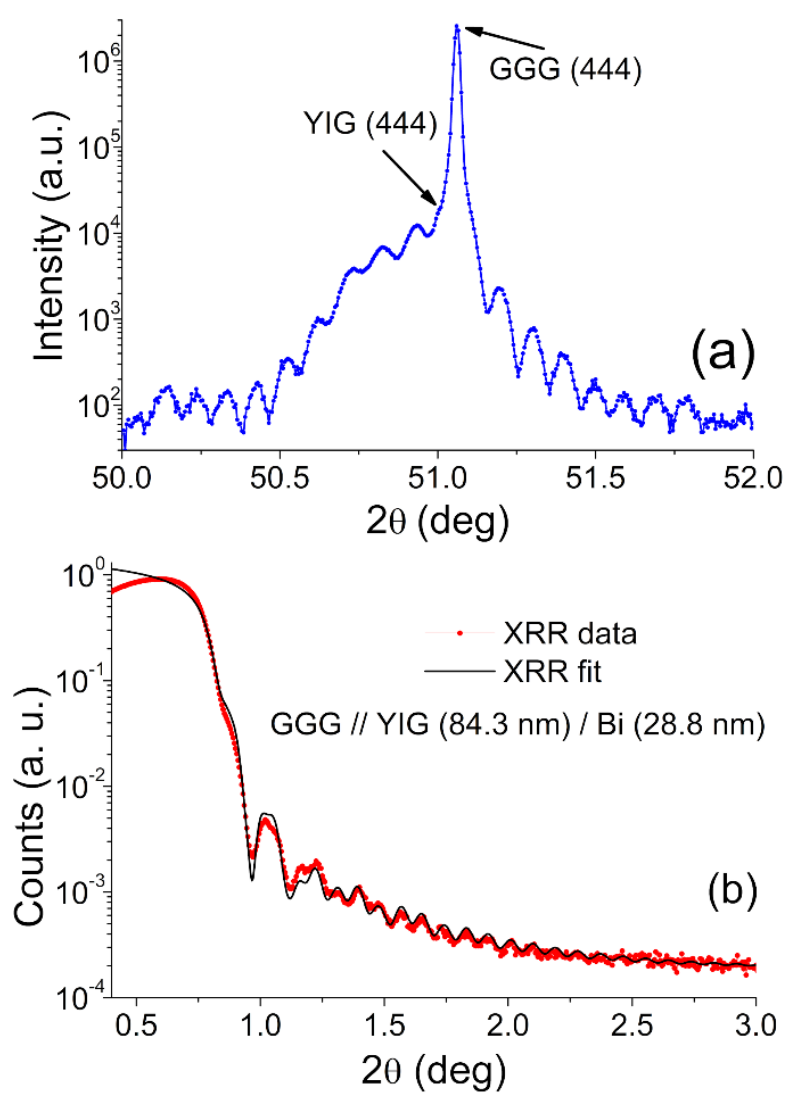

Figure 2. (a) XRD pattern of a YIG thin film grown by PLD on a GGG(111) substrate around the (444) Bragg peak of GGG and YIG. (b) XRR measurement of a YIG (84.3 $\mathrm{nm}) / \mathrm{Bi}(28.8 \mathrm{~nm})$ stack. Black solid line is the numerical analysis of the XRR data by means of the commercial software Leptos provided by Bruker, which allows us to obtain the thickness of each layer.

We started the study of the magnetization dynamic properties by performing FMR on GGG // YIG thin films at different excitation frequencies. Fitting the dependence of the resonance magnetic field on the frequency to the Kittel equation (see figure 3(a)), the gyromagnetic ratio and the saturation magnetization of the YIG thin films are found to be $\gamma_{Y I G}=1.80 \times 10^{11} \frac{\mathrm{rad}}{\mathrm{s} \cdot \mathrm{T}}$ and $M_{S, Y I G}=1.577 \times 10^{5} \frac{\mathrm{A}}{\mathrm{m}}=157.7 \mathrm{emu} / \mathrm{cm}^{3}$, very close to the bulk YIG values $\left(\gamma_{\text {bulk } Y I G}=1.76 \times 10^{11} \frac{\mathrm{rad}}{\mathrm{s} \cdot T}\right.$ and $\left.M_{S \text {, bulk } Y I G}=140 \mathrm{emu} / \mathrm{cm}^{3}\right)$ [19]. Regarding the linewidth of the FMR peaks, if we analyze its dependence on the excitation frequency we can obtain the Gilbert damping parameter [20]. We obtain, by fitting to a linear dependence the variation of the linewidth with the excitation frequency shown in figure 3(b), a value of $\alpha_{Y I G}=2.4(2) \times 10^{-3}$, that is within the values reported for YIG films grown by PLD [20-24], confirming the suitability of the YIG thin films we have deposited for spin pumping experiments. 

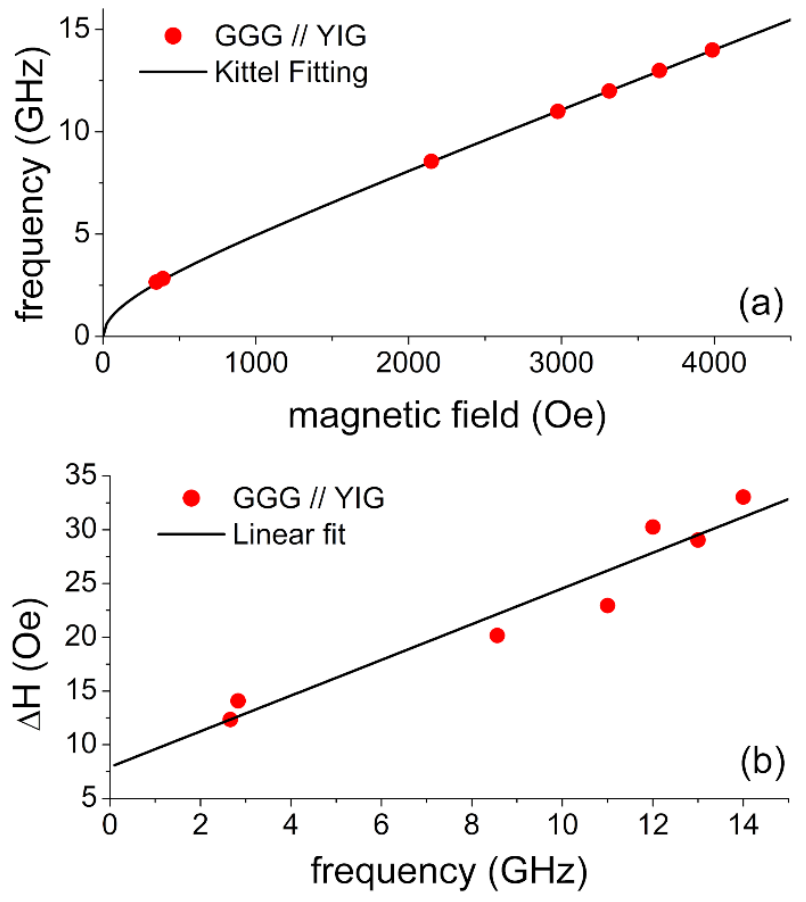

Figure 3. (a) Frequency as a function of resonance magnetic field for a GGG // YIG (84.3 $\mathrm{nm}$ ) thin film. Solid line is a fitted curve to the Kittel equation. (b) Full FMR linewidth as a function of resonance frequency for a GGG // YIG $(84.3 \mathrm{~nm})$ thin film. Solid line is the linear fitting of the data.

When measuring the magnetization dynamic properties of YIG/Bi stacks, the excitation of the FMR of the YIG layer will inject a spin current from the ferromagnetic YIG layer into the Bi layer due to spin pumping. This spin current will be converted into a dc voltage signal by ISHE. The frequency dependence of the ISHE voltage signal measured for a $\mathrm{YIG} / \mathrm{Bi}(\mathrm{t})$ stack with a thickness of the $\mathrm{Bi}$ layer $\mathrm{t}=14.1 \mathrm{~nm}$ is shown in figure 4(a). The dependence of the frequency on the static resonance magnetic field is presented in figure $4(b)$, where the solid line is the fitted curve to the Kittel equation. 


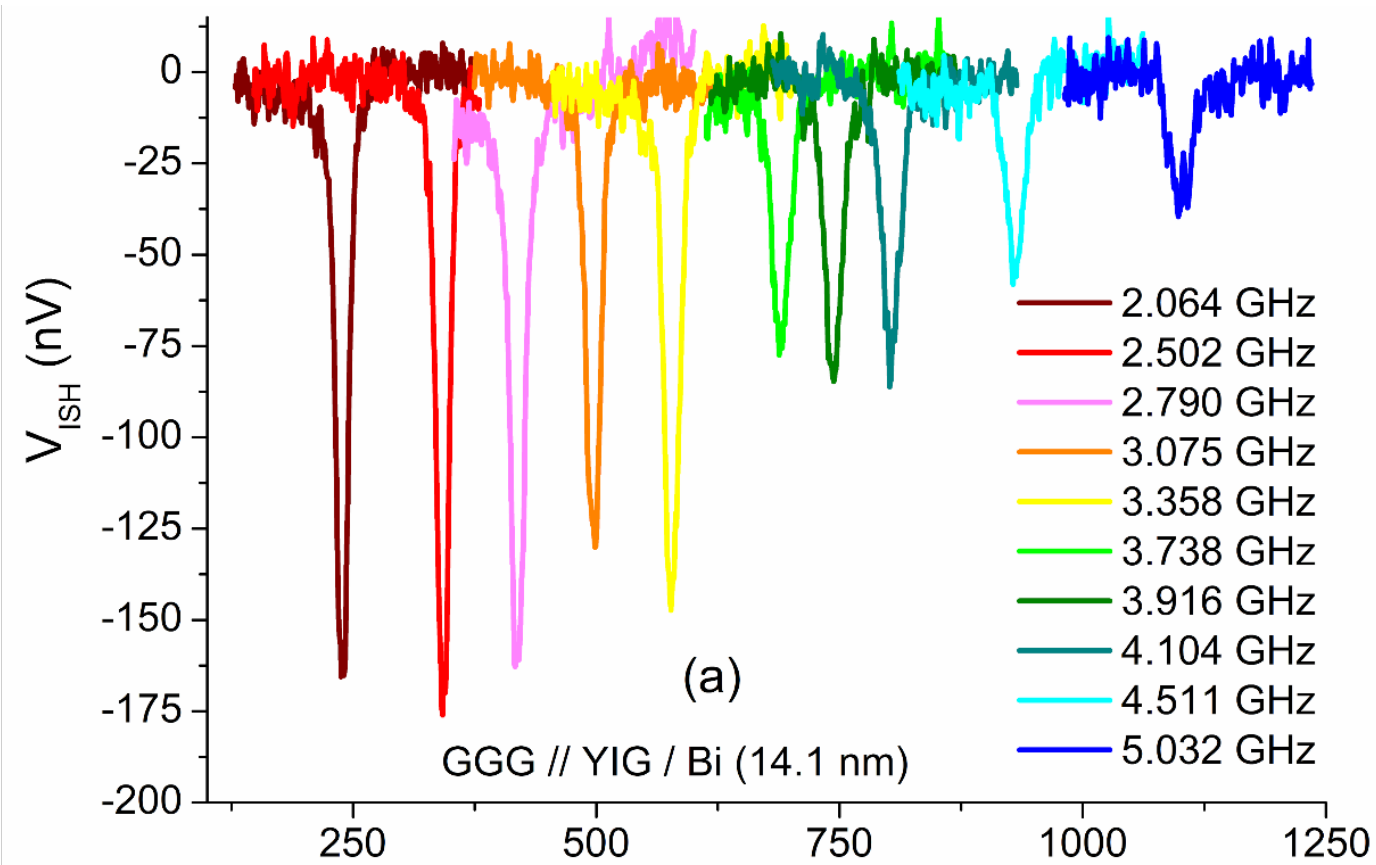

magnetic field $(\mathrm{Oe})$
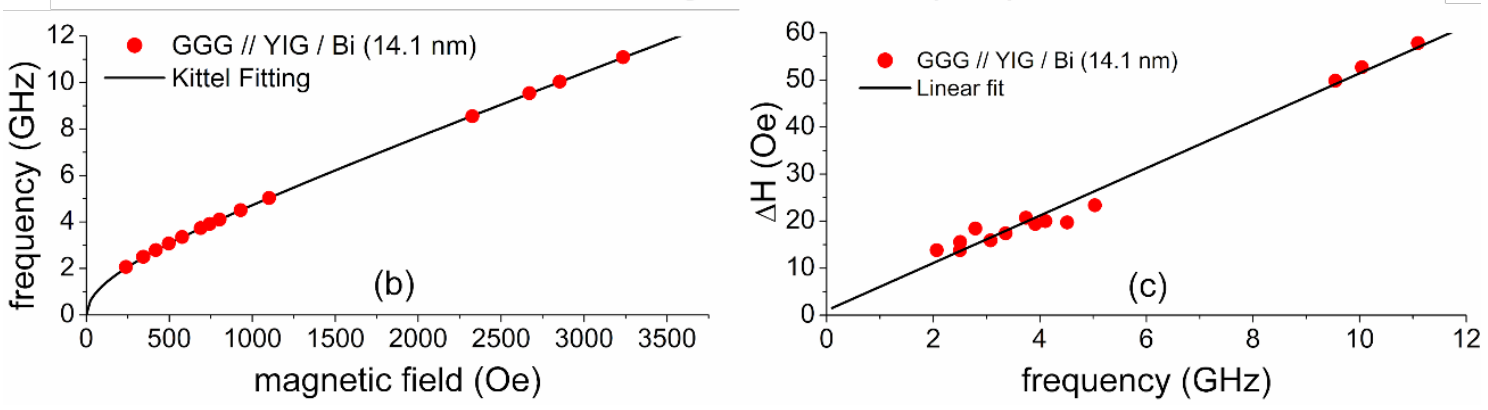

Figure 4. (a) Inverse spin Hall voltage as a function of magnetic field using an if power of $10 \mathrm{dBm}$ for a GGG // YIG / Bi (14.1 nm) stack at different frequencies between 2 and $6 \mathrm{GHz}$. (b) Frequency as a function of resonance magnetic field for a GGG // YIG / Bi $(14.1 \mathrm{~nm})$ stack. Solid line is a fitted curve to the Kittel equation. (c) Full FMR linewidth as a function of resonance frequency for a GGG // YIG / Bi (14.1 nm) stack. Solid line is the linear fitting of the data.

As we have previously discussed for YIG thin films, we can study, both the position and the linewidth of the ISHE voltage signal as a function of frequency and obtain the magnetic properties of YIG and also the damping of the YIG / Bi samples. In figure 4(c) the frequency dependence of the full FMR linewidth for a YIG / Bi(t) stack with $t=14.1$ $\mathrm{nm}$ is displayed. By fitting this dependence to a linear relation, we obtain a Gilbert damping parameter that is enhanced with respect to the value found in YIG thin films: $\boldsymbol{\alpha}_{Y I G / B i(14.1 \mathrm{~nm})}=6.7(2) \times 10^{-3}$. Damping is enhanced in thin magnetic films with nonmagnetic adjacent layers by the spin-pumping effect through the interface. The pumping of spins by the magnetic layer into the adjacent non-magnetic layer, slows down the precession of the magnetization and this corresponds to an enhanced Gilbert damping constant [25]. From the magnitude of this damping enhancement we can calculate the spin mixing conductance of the YIG / Bi interface and we obtain a value: 


$$
g_{e f f}^{\uparrow \downarrow}=\frac{\left(\alpha_{\frac{Y I G}{B i}}-\alpha_{Y I G}\right)}{g \mu_{B}} 4 \pi M_{S} t_{Y I G}=4.0(3) \times 10^{18} \mathrm{~m}^{-2}
$$

The spin-mixing conductance is directly related to the transparency of the $\mathrm{YIG} / \mathrm{Bi}$ interface for transfer of spin angular momentum across it. The value we have obtained for $g_{e f f}^{\uparrow \downarrow}, 4.0(3) \times 10^{18} \mathrm{~m}^{-2}$, is about three times higher than the value reported previously for the YIG/Bi interface (see supplementary material in ref. [26]). Based on the spinmixing conductance we can calculate the spin mixing conductivity, $G_{e f f}^{\uparrow \downarrow}$, by multiplying by the von Klitzing conductance as $G_{e f f}^{\uparrow \downarrow}=\left(e^{2} / h\right) g_{e f f}^{\uparrow \downarrow}$ [27], and we obtain a value of $G_{e f f}^{\uparrow \downarrow}=1.6(1) \times 10^{14} \Omega^{-1} m^{-2}$, similar to the values reported for YIG/AI [28], YIG/Ta and YIG/Pt interfaces [29].

We have also performed ISHE voltage measurements on YIG/Bi stacks containing different bismuth thicknesses in the range between 10 and 50 nanometers. The ISHE voltage peaks measured at a frequency of $2.5 \mathrm{GHz}$ are displayed in figure $5(\mathrm{a})$.

By fitting these peaks to Lorentzian profiles, we obtain the height of the ISHE voltage as a function of bismuth thickness, which is shown in figure 5(b). The ISHE voltage signal increases almost linearly until it reaches its maximum for a bismuth thickness around 25 $\mathrm{nm}$ followed by a decrease for larger thicknesses.
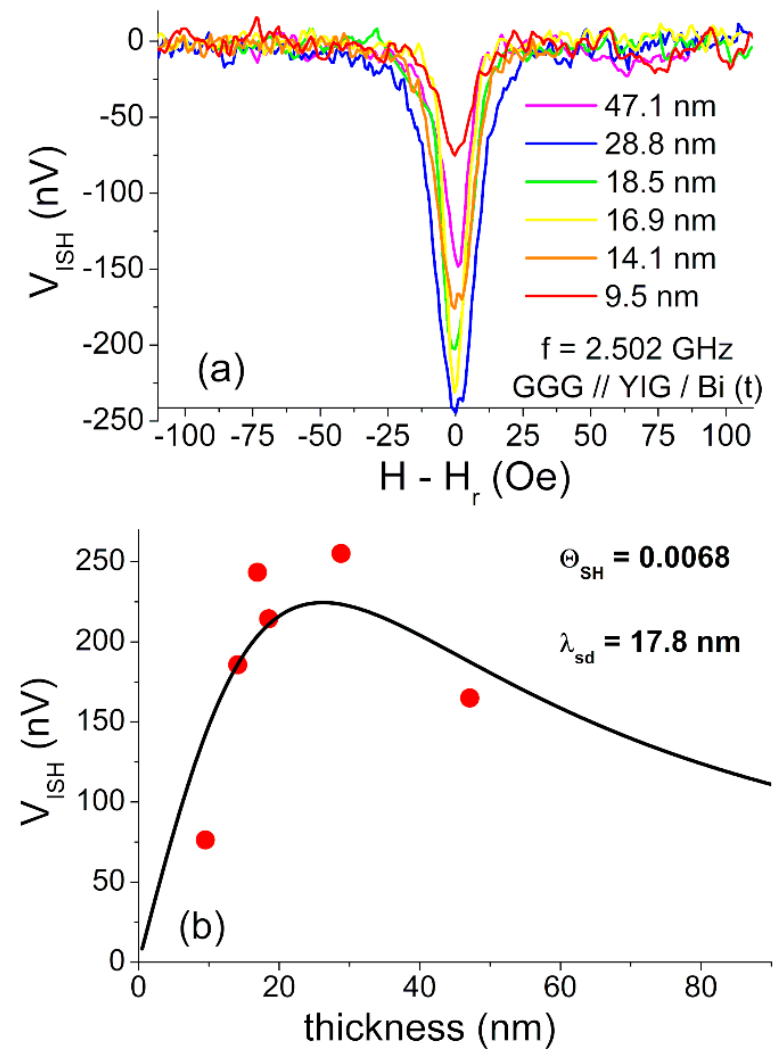

Figure 5. (a) ISHE voltage measured in GGG//YIG $(85 \mathrm{~nm}) / \mathrm{Bi}$ stacks with different $\mathrm{Bi}$ thicknesses. Excitation frequency and power are $2.502 \mathrm{GHz}$ and $10 \mathrm{dBm}$, respectively. (b) Dependence of the ISHE voltage signal on Bi thickness for a microwave frequency of $2.502 \mathrm{GHz}$. Solid line is the fitting curve derived from equation (2) (using as input 
parameters $\mathrm{f}=2.502 \mathrm{GHz}, \mathrm{L}=1 \mathrm{~mm}, \mathrm{P}=1.157$ and $\theta=0.93 \mathrm{deg})$, for $\Theta_{\mathrm{SH}}=0.0068(8)$ and $\lambda_{\mathrm{sd}}=17.8(9) \mathrm{nm}$.

This dependence of the ISHE voltage signal on the non-magnetic material thickness is the expected one when considering the spin-diffusion equation with the appropriate experimental boundary conditions [29,30]:

$$
V_{I S H}=\Theta_{S H} \frac{G_{e f f}^{\uparrow \downarrow}}{G_{e f f}^{\uparrow \downarrow}+\frac{\sigma}{\lambda_{s d}} \frac{1-\exp \left(-2 t_{B i} / \lambda_{s d}\right)}{1+\exp \left(-2 t_{B i} / \lambda_{s d}\right)}} \frac{h L P f \sin ^{2} \theta}{2 e t_{B i}} \frac{\left[1-\exp \left(-t_{B i} / \lambda_{s d}\right)\right]^{2}}{1+\exp \left(-2 t_{B i} / \lambda_{s d}\right)}
$$

Where $\Theta_{S H}$ is the spin Hall angle, characterizing the efficiency of the spin to charge conversion in the bismuth layer, $\sigma$ is the conductivity of the bismuth layer and $t_{B i}$ is its thickness, $\lambda_{s d}$ is the spin-diffusion length in bismuth, which characterizes the decay of the injected spin current in the Bi layer, $L$ is the length of the YIG/Bi stack being excited by the microwave field $(1 \mathrm{~mm})$ at a frequency $f, \theta$ is the angle of magnetization precession in the YIG layer and $P$ is the ellipticity correction factor [31].

We can estimate the angle of magnetization precession in the YIG layer by taking into account the geometry of the shorted if line and the full FMR linewidth of the YIG films (figure 3(b)). When exciting with a microwave output power of $10 \mathrm{dBm}$ the calculated microwave field is $0.099 \mathrm{Oe}$ and the corresponding angle of magnetization precession at a frequency of $2.5 \mathrm{GHz}$ is $0.93 \mathrm{deg}$. Therefore, we can fit the Bi thickness dependence of the ISHE voltage signal to the expression provided by equation (2) with only two free parameters, the spin hall angle, $\Theta_{S H}$ and the spin-diffusion length, $\lambda_{s d}$. We obtain a spin Hall angle of $0.0068(8)$, which implies an efficiency of $0.68 \%$ for spin-to-charge conversion in bismuth, which is comparable to the values reported in several high spinorbit coupling materials such as bismuth [7-9], platinum [15] or tantalum [29]. In addition, the spin-diffusion length obtained from the fitting described above is $17.8(9) \mathrm{nm}$, which is very similar to the value found for bismuth thin films grown in the same laboratory [9], and larger than most of values reported for platinum [15].

Finally, we have investigated the evolution of the ISHE voltage signal with the aging of YIG / Bi samples, which is of immediate relevance when considering a practical implementation in spintronic devices. For this purpose, we have stored the samples at ambient conditions and we have measured their ISHE voltage signal during the following days (up to 150 days) to track the time evolution of the ISHE voltage signal. We have obtained a continuous decrease in magnitude and a slight broadening of the ISHE voltage peaks as a consequence of exposing the samples to ambient conditions. As can be seen in figure 6 , we have found that for $\mathrm{Bi}$ thicknesses lower or similar to the spindiffusion length in bismuth of about $18 \mathrm{~nm}$, the ISHE voltage signal is still above $80 \%$ of its initial value after 100 days. On the contrary, in samples with Bi thicknesses well above the spin-diffusion length, the voltage signal is already below $80 \%$ of its initial value after 50 days. Our results indicate that bismuth thicknesses around or below the spin-diffusion length should be used in practical implementation of YIG / Bi samples to limit the impact of sample degradation on the spin-to-charge conversion efficiency. Otherwise, we 
suggest encapsulating the samples to minimize the effects of the exposure to ambient conditions.
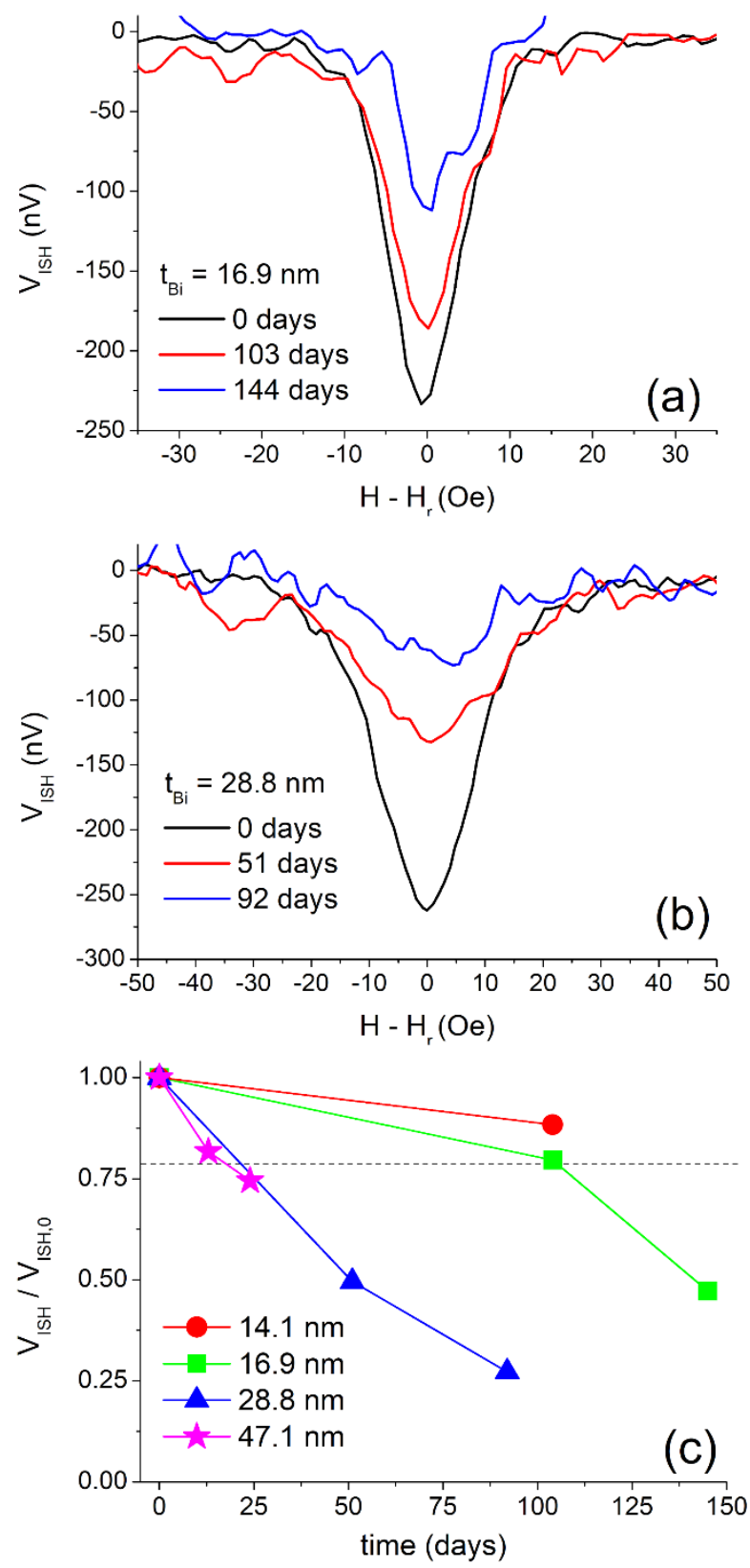

Figure 6. (a) ISHE voltage measured in a GGG // YIG / Bi (16.9 nm) stack after being stored at ambient conditions for different time periods. (b) ISHE voltage measured in a GGG // YIG / Bi (28.8 nm) stack after being stored at ambient conditions for different time periods. (c) Ratio of the ISHE voltage signal for GGG // YIG / Bi stacks over the initial signal, as a function of the time for the samples (stored at ambient conditions). Dashed line represents the $80 \%$ value. Solid lines are guide to the eyes. Excitation frequency and power are $2.502 \mathrm{GHz}$ and $10 \mathrm{dBm}$, respectively. 


\section{Conclusion}

In summary, we have optimized the growth of GGG // YIG / Bi stacks and determined, from measurements of the ISHE voltage signal in spin pumping experiments, the values for the spin Hall angle and the spin-diffusion length in bismuth, $\Theta_{S H}=0.0068(8)$ and $\lambda_{s d}=17.8(9) \mathrm{nm}$, in good agreement with previous reports of spin-to-charge conversion in $\mathrm{Bi}$ [7-9]. We have also studied the influence of aging on the ISHE voltage signal, finding that in samples with $\mathrm{Bi}$ thicknesses around or below the spin-diffusion length the voltage signal is still above $80 \%$ of its initial value after storing the samples in ambient conditions for 100 days. The optimization of the growth of YIG / Bi stacks and the study of the influence of aging on the spin-to-charge conversion efficiency reported in this work lay the foundations for the design of spintronic devices that mitigate the influence of aging on the ISHE voltage signal by choosing the proper thickness of the non-magnetic layer.

\section{Acknowledgments}

Authors acknowledge financial support from the Spanish Ministry of Economy and Competitiveness through the projects MAT2017-82970-C2-1-R and MAT2017-82970C2-2-R, from the University of Zaragoza through the project JIUZ-2019-CIE-08 and from the Aragon Regional Government (Construyendo Europa desde Aragón) through the project E13_20R, with the European Social Fund funding.

\section{Data availability statement}

The data that support the findings of this study are available upon reasonable request from the authors.

\section{REFERENCES}

[1] Puebla J, Kim J, Kondou K and Otani Y 2020 Spintronic devices for energyefficient data storage and energy harvesting Commun. Mater. 124

[2] Brataas A, van Wees B, Klein O, de Loubens G and Viret M 2020 Spin insulatronics Phys. Rep. 885 1-27

[3] Dieny B, Prejbeanu I L, Garello K, Gambardella P, Freitas P, Lehndorff R, Raberg W, Ebels U, Demokritov S O, Akerman J, Deac A, Pirro P, Adelmann C, Anane A, Chumak A V., Hirohata A, Mangin S, Valenzuela S O, Onbaşlı M C, d'Aquino M, Prenat G, Finocchio G, Lopez-Diaz L, Chantrell R, ChubykaloFesenko $O$ and Bortolotti P 2020 Opportunities and challenges for spintronics in the microelectronics industry Nat. Electron. 3 446-59

[4] Sinova J, Valenzuela S O, Wunderlich J, Back C H and Jungwirth T 2015 Spin Hall effects Rev. Mod. Phys. 87 1213-60

[5] Saitoh E, Ueda M, Miyajima H and Tatara G 2006 Conversion of spin current 
into charge current at room temperature: Inverse spin-Hall effect Appl. Phys. Lett. 88182509

[6] Valenzuela S O and Tinkham M 2006 Direct electronic measurement of the spin Hall effect Nature 442 176-9

[7] Hou D, Qiu Z, Harii K, Kajiwara Y, Uchida K, Fujikawa Y, Nakayama H, Yoshino T, An T, Ando K, Jin X and Saitoh E 2012 Interface induced inverse spin Hall effect in bismuth/permalloy bilayer Appl. Phys. Lett. 101042403

[8] Emoto H, Ando Y, Shikoh E, Fuseya Y, Shinjo T and Shiraishi M 2014 Conversion of pure spin current to charge current in amorphous bismuth J. Appl. Phys. 11517 C507

[9] Sangiao S, De Teresa J M, Morellon L, Lucas I, Martinez-Velarte M C and Viret M 2015 Control of the spin to charge conversion using the inverse RashbaEdelstein effect Appl. Phys. Lett. 106172403

[10] Yue D, Lin W, Li J, Jin X and Chien C L 2018 Spin-to-Charge Conversion in Bi Films and Bi/Ag Bilayers Phys. Rev. Lett. 121037201

[11] Mallmann E J J, Sombra A S B, Goes J C and Fechine P B A 2013 Yttrium iron garnet: Properties and applications review Solid State Phenom. 202 65-96

[12] Althammer M 2018 Pure spin currents in magnetically ordered insulator/normal metal heterostructures J. Phys. D-Applied Phys. 51313001

[13] Azevedo A, Vilela Leão L H, Rodriguez-Suarez R L, Oliveira A B and Rezende $S$ M 2005 Dc effect in ferromagnetic resonance: Evidence of the spin-pumping effect? J. Appl. Phys. 97 10C715

[14] Zhang W, Vlaminck V, Pearson J E, Divan R, Bader S D and Hoffmann A 2013 Determination of the Pt spin diffusion length by spin-pumping and spin Hall effect Appl. Phys. Lett. 103242414

[15] Rojas-Sánchez J C, Reyren N, Laczkowski P, Savero W, Attané J P, Deranlot C, Jamet M, George J M, Vila L and Jaffrès H 2014 Spin pumping and inverse spin hall effect in platinum: The essential role of spin-memory loss at metallic interfaces Phys. Rev. Lett. 112106602

[16] Santana F A F, Da Silva J M, Vasconcelos T C, Ramos J G G S and Barbosa A L R 2020 Spin Hall angle fluctuations in a device with disorder Phys. Rev. B 102 041107

[17] Marcano N, Sangiao S, Magén C, Morellón L, Ibarra M R, Plaza M, Pérez L and De Teresa J M 2010 Role of the surface states in the magnetotransport properties of ultrathin bismuth films Phys. Rev. B - Condens. Matter Mater. Phys. 82125326

[18] Marcano N, Sangiao S, De Teresa J M, Morellón L, Ibarra M R, Plaza M and Pérez L 2010 Structural and magnetotransport properties of Bi thin films grown by thermal evaporation J. Magn. Magn. Mater. 3221460

[19] Stancil, D. D. and Prabhakar A 2009 Spin Waves - Theory and applications ed Springer (Springer New York)

[20] Jungfleisch M B, Chumak A V., Kehlberger A, Lauer V, Kim D H, Onbasli M C, Ross C A, Kläui M and Hillebrands B 2015 Thickness and power dependence of 
the spin-pumping effect in $\mathrm{Y} 3 \mathrm{Fe} 5 \mathrm{O} 12 / \mathrm{Pt}$ heterostructures measured by the inverse spin Hall effect Phys. Rev. B - Condens. Matter Mater. Phys. 91134407

[21] D'Allivy Kelly O, Anane A, Bernard R, Ben Youssef J, Hahn C, Molpeceres A H, Carrétéro C, Jacquet E, Deranlot C, Bortolotti P, Lebourgeois R, Mage J C, De Loubens G, Klein O, Cros V and Fert A 2013 Inverse spin Hall effect in nanometer-thick yttrium iron garnet/Pt system Appl. Phys. Lett. 103082408

[22] Onbasli M C, Kehlberger A, Kim D H, Jakob G, Kläui M, Chumak A V., Hillebrands B and Ross C A 2014 Pulsed laser deposition of epitaxial yttrium iron garnet films with low Gilbert damping and bulk-like magnetization $A P L$ Mater. 2106102

[23] Haertinger M, Back C H, Lotze J, Weiler M, Geprägs S, Huebl H, Goennenwein S T B and Woltersdorf G 2015 Spin pumping in YIG/Pt bilayers as a function of layer thickness Phys. Rev. B - Condens. Matter Mater. Phys. 92054437

[24] Ruiz-Gómez S, Serrano A, Guerrero R, Muñoz M, Lucas I, Foerster M, Aballe L, Marco J F, Amado M, McKenzie-Sell L, Di Bernardo A, Robinson J W A, González Barrio M Á, Mascaraque A and Pérez L 2018 Highly Bi-doped Cu thin films with large spin-mixing conductance APL Mater. 6101107

[25] Tserkovnyak Y and Brataas A 2002 Enhanced Gilbert Damping in Thin Ferromagnetic Films Phys. Rev. Lett. 88117601

[26] Emoto H, Ando Y, Eguchi G, Ohshima R, Shikoh E, Fuseya Y, Shinjo T and Shiraishi M 2016 Transport and spin conversion of multicarriers in semimetal bismuth Phys. Rev. B - Condens. Matter Mater. Phys. 93174428

[27] Boone C T, Nembach H T, Shaw J M and Silva T J 2013 Spin transport parameters in metallic multilayers determined by ferromagnetic resonance measurements of spin-pumping J. Appl. Phys. 113153906

[28] Das K S, Dejene F K, Van Wees B J and Vera-Marun I J 2019 Temperature dependence of the effective spin-mixing conductance probed with lateral nonlocal spin valves Appl. Phys. Lett. 114072405

[29] Hahn C, de Loubens G, Klein O, Viret M, Naletov V V and Ben Youssef J 2013 Comparative measurements of inverse spin Hall effects and magnetoresistance in YIG/Pt and YIG/Ta Phys. Rev. B 87174417

[30] Castel V, Vlietstra N, Ben Youssef J and Van Wees B J 2012 Platinum thickness dependence of the inverse spin-Hall voltage from spin pumping in a hybrid yttrium iron garnet/platinum system Appl. Phys. Lett. 101132414

[31] Ando K, Yoshino T and Saitoh E 2009 Optimum condition for spin-current generation from magnetization precession in thin film systems Appl. Phys. Lett. 94152509 